\title{
Papers
}

\section{Effect of iron supplementation on incidence of infectious illness in children: systematic review}

Tarun Gera, H P S Sachdev

\begin{abstract}
Objective To evaluate the effect of iron supplementation on the incidence of infections in children.

Design Systematic review of randomised controlled trials.

Data sources 28 randomised controlled trials (six unpublished and 22 published) on 7892 children. Interventions Oral or parenteral iron supplementation or fortified formula milk or cereals. Outcomes Incidence of all recorded infectious illnesses, and individual illnesses, including respiratory tract infection, diarrhoea, malaria, other infections, and prevalence of positive smear results for malaria. Results The pooled estimate (random effects model) of the incidence rate ratio (iron $v$ placebo) was 1.02 (95\% confidence interval 0.96 to $1.08, \mathrm{P}=0.54$; $\mathrm{P}<0.0001$ for heterogeneity). The incidence rate difference (iron minus placebo) for all recorded illnesses was 0.06 episodes/child year $(-0.06$ to 0.18 , $\mathrm{P}=0.34 ; \mathrm{P}<0.0001$ for heterogeneity). However, there was an increase in the risk of developing diarrhoea (incidence rate ratio $1.11,1.01$ to $1.23, \mathrm{P}=0.04$ ), but this would not have an overall important on public health (incidence rate difference 0.05 episodes/child year, -0.03 to $0.13 ; \mathrm{P}=0.21$ ). The occurrence of other illnesses and positive results on malaria smears (adjusted for positive smears at baseline) were not significantly affected by iron administration. On meta-regression, the statistical heterogeneity could not be explained by the variables studied.

Conclusion Iron supplementation has no apparent harmful effect on the overall incidence of infectious illnesses in children, though it slightly increases the risk of developing diarrhoea.
\end{abstract}

\section{Introduction}

Anaemia caused by iron deficiency is a major public health problem, affecting $46 \%$ of school children globally. Iron deficiency has adverse effects on psychomotor development ${ }^{2}$ and on the capacity to work. The reversible consequences in childhood have prompted recommendations for early intervention. The proposed interventions rely primarily on enhancing iron intake either through supplementation or fortification of food. ${ }^{34}$
Because of these proposed interventions their safety needs to be unequivocally established. The role of iron in resistance to disease remains controversial. Iron deficiency may be an important defence mechanism, and the term "nutritional immunity" was coined to highlight the importance of hypoferraemia in preventing bacterial growth. ${ }^{5}$ Conversely, data suggest that iron deficiency is associated with impairment of cell mediated immunity and the bactericidal activity of neutrophils, thus increasing susceptibility to infection. ${ }^{6}$ Iron supplementation may also cause damage to cells mediated through free radicals. ${ }^{8}$ Objective safety data from longitudinal studies of iron supplementation are conflicting; trials have shown either beneficial effects, ${ }^{9}$ no effect, ${ }^{10}$ or an increase in infectious illnesses. ${ }^{11}{ }^{12}$ Children, particularly infants and those living in developing countries, are vulnerable to infectious diseases. It is thus important to establish the safety of iron supplementation in children on a public health scale. We conducted a systematic review to determine the effect of iron supplementation on infectious illnesses.

\section{Methods}

\section{Inclusion criteria}

To be included trials had to be randomised placebo controlled trials-except for those in which iron was given parenterally, in which case trials could be non-placebo controlled because it would be difficult to administer a similar placebo; had to investigate iron supplementation through the oral or the parenteral route or as formula milk or cereals fortified with iron; and evaluate one or more infectious illnesses as an outcome measure. We also included studies in which other micronutrients and drugs were simultaneously administered if the only difference between the study and the control groups was iron supplementation.

\section{Data collection}

We searched computerised bibliographic medical databases, including Medline, Cochrane controlled trials register, Embase, IBIDS, and Healthstar. We also reviewed reference lists of identified articles and hand searched reviews, bibliographies of books, and abstracts and proceedings of international conferences or meetings. Donor agencies, "experts," and authors of recent iron supplementation trials were contacted to identify any additional or ongoing trials. The title and \begin{tabular}{l}
\hline $\begin{array}{l}\text { Editorial by } \\
\text { Tomkins }\end{array}$ \\
\hline \\
Division of Clinical \\
Epidemiology, \\
Department of \\
Paediatrics, \\
Maulana Azad \\
Medical College, \\
New Delhi 110002, \\
India \\
Tarun Gera \\
senior resident \\
H P S Sachdev \\
professor \\
Correspondence to: \\
H P S Sachdev \\
E-6/12, Vasant \\
Vihar, New Dehli \\
110057, India \\
hpssachdev@ \\
hotmail.com \\
bmj.com 2002;325:1142
\end{tabular} 
abstract of the studies identified in the computerised search were scanned to exclude studies that were obviously irrelevant. We retrieved the full text of the remaining studies and identified studies that fulfilled the inclusion criteria. To avoid publication bias we included published and unpublished trials.

\section{Quality of methods}

We assessed the quality of trials using recommended criteria. ${ }^{13}{ }^{14}$ Concealment of allocation was classed as adequate, unclear, inadequate, or not used. To assess completeness of follow up we classified studies by percentage of participants excluded $(<3 \%, 3-9.9 \%$, $10-19.9 \%$, and $\geqslant 20 \%)$. Blinding was classified as double blinding, single blinding, no blinding, and unclear. TG Abstracted all data.

\section{Data abstraction}

We used preformed questionnaires to abstract data. The data included in this review were derived from the published papers or were provided by the authors. Illnesses and the outcomes included were as defined by the authors. Whenever possible we contacted the authors for clarifications.

\section{Statistical analysis}

The presence of bias in the extracted data was evaluated by funnel plots. ${ }^{15}$ We used the metabias command in Stata software to perform the statistical tests for funnel plot asymmetry. ${ }^{16}$ The pooled estimates of incidence rate ratio and incidence rate difference were calculated by StatsDirect statistical software (version 1.9.5; StatsDirect, Cambridge) with fixed effects and random effects model assumptions. ${ }^{17}$ This program also computes the formal test of heterogeneity ( $Q$ statistic). We primarily report random effects estimates because most of the pooled results obtained were statistically heterogeneous. We chose incidence rate summary to account for the differences in duration of follow up in the various extracted studies. The data were recorded in the form of the total number of episodes of illness and the person time exposed (in child years). For trials in which the results were available in this format we recorded the figures directly from the publication, and this category of studies was labelled as the "actual" group. In the "computed" group of trials, the person time of follow up was not provided, and we calculated estimates from the product of the duration of follow up and the sample sizes available at the

Table 1 Characteristics of excluded trials

Reason for exclusion

\begin{tabular}{|c|c|}
\hline & Reason for exclusion \\
\hline Andelman $^{18}$ & Information not extractable \\
\hline Burman $^{19}$ & Information not extractable \\
\hline Damodaran $^{20}$ & Supplemented folic acid with iron \\
\hline Oppenheimer $^{21}$ & Same study group in included trial \\
\hline Bates $^{22}$ & Supplemented other micronutrients \\
\hline Heresi ${ }^{23}$ & Supplemented other micronutrients \\
\hline Chwang $^{9}$ & Information not extractable \\
\hline Heywood ${ }^{24}$ & Subset of an included trial \\
\hline Angeles-Agdeppa ${ }^{25}$ & Supplemented other micronutrients \\
\hline Heresi $^{26}$ & Supplemented other micronutrients \\
\hline Van Hensbroek $^{27}$ & Supplemented multiple antimalarials in crossover manner \\
\hline Beck $^{28}$ & Same study group in an included trial \\
\hline Von Stujvenberg $^{29}$ & Supplemented other micronutrients \\
\hline Picaud $^{30}$ & Erythropoietin given with iron supplementation \\
\hline Allen, Lonnerdal, Ninh, Zimmermann, Quyen & Unpublished, full text not available from authors \\
\hline
\end{tabular}

beginning and the end of the study. In some trials data were obtained by quantitative analysis of published graphs.

Some studies had reported only on the prevalence of malaria parasitaemia confirmed from smears at the beginning and the end of the supplementation period. Pooled estimates of the odds ratio of positive smears at the end of the supplementation period were computed by the "meta" command in Stata software. ${ }^{16}$ We also performed a meta-regression (restricted maximum likelihood iteration) through the "metareg" command in Stata software to determine the pooled log odds ratio of developing malaria in the group with iron supplementation compared with the placebo group. The covariate in the meta-regression equation was the log odds ratio at the beginning of the trial to adjust for the baseline differences in the prevalence of malaria.

We carried out stratified analyses for quality of methods; case detection (active field based or passive facility based); specificity of case definition; route of iron administration (parenteral, oral supplement, or fortified food); dose-this was initially planned but could not be performed as it could not be extracted for each study; duration of supplementation; type of illness (gastrointestinal, respiratory, malaria, non-diarrhoeal, or others); and baseline haemoglobin concentration in the supplemented group. The contribution of these variables to heterogeneity was also explored by meta-regression. ${ }^{16}{ }^{17}$

\section{Results}

We identified 47 randomised controlled trials that were potentially eligible. Of these, 38 trials were published in medical journals or were theses ${ }^{9-52}$ and 9 were unpublished (box 1). Nineteen studies were ineligible (table 1). We therefore evaluated 28 studies (22 published $^{101131-4447-52}$ two theses, ${ }^{4546}$ and six unpublished) in this systematic review.

\section{Baseline characteristics of the studies}

Table 2 depicts the baseline characteristics of the included trials. Thirteen trials were in children aged $<1$ year, 10 studies included preschool children $(\leqslant 5$ years), and five trials included children aged $>5$ years. Eleven trials were from Africa, eight from Asia, five from the Americas, two from Europe, and two from Australia and New Zealand. The eligibility and exclusion criteria varied. Most of the studies used oral iron supplementation (20/28; 71\%). Three trials used parenteral administration, and five studies used iron fortified foods.

Differences in the mode of administration may have implications for bioavailability of iron and its possible effect on the immune function. The supplementation dose used could influence the degree to which illness was affected. As a crude generalisation, the fortified formulas had the lowest dosage and the parenteral route had the highest. The duration of supplementation and follow up for oral intake varied from 2 months to 30 months.

The specificity of the definition used for illness was variable. Specificity of diagnosis has the potential to bias the observed effect of supplementation on illness. For example, low specificity definitions could underestimate the effect of iron supplementation on malaria 


\section{Unpublished studies}

Papers presented at International Nutritional Anaemia Consultative Group (INACG) Symposium, Hanoi, Vietnam, 2001

Allen LH, Lopez P, Galvaz IA, Garcia DP, Isoard F, Rosado JL. Does multiple micronutrient

supplementation increase haemoglobin and iron status more than iron alone?

Lonnerdal B, Domellof M, Dewey KG, Cohen R, Rivera LL, Hernell O. Effects of iron supplementation of breastfed infants in Honduras and Sweden from 4-9 or 6-9 months of age.

Ninh NX, Berger J, Tolvanen M, Trung NQ, Nhien NV,

Lien DK, et al. Control of iron deficiency anaemia in

Vietnamese infants by efficacy of iron and zinc supplementation to reduce anaemia and growth faltering in Vietnamese infants.

Zimmermann M, Hess S, Adou P, Torresani T, Cook J,

Hurrell R. Treatment of iron deficiency in goitrous children improves the efficacy of iodized salt.

Quyen DT, Berger J, Ninh NX, Khan NC, Khoi HH.

Control of iron deficiency anaemia in Vietnamese infants by weekly and daily iron supplementation: efficacy and effectiveness.

Atukorala S, de Silva A, Ahluwalia N. Evaluation of iron status of children in the presence of infections: effect of iron supplementation on iron status, infection and morbidity.*

\section{Other unpublished papers}

Rice AL, Stoltzfus RJ, Tielsch JM, Savioli L, Montresor A, Albonico M, et al. Iron supplementation and mebendazole treatment do not affect respiratory or diarrhoeal morbidity incidence rates in Tanzanian preschoolers. 1999*

Agarwal D, Sachdev HPS, Mallika V, Singh T. Iron supplementation in breast fed, full term, low birth weight infants. 1999.*

Nagpal J, Sachdev HPS, Mallika V, Singh T. Iron supplementation with complementary feeding in predominantly breastfed infants. 2000*

*Included in the review

due to a high rate of misclassification of non-malarial fevers as malaria. In some studies, fever was recorded as an additional infectious illness because fever in children is mostly attributed to infectious diseases. ${ }^{41}{ }^{51}$ Inclusion of fever as a separate infection may lead to duplication of data because fever may accompany malaria, respiratory tract infection, and diarrhoea. However, we have included it on the assumption that

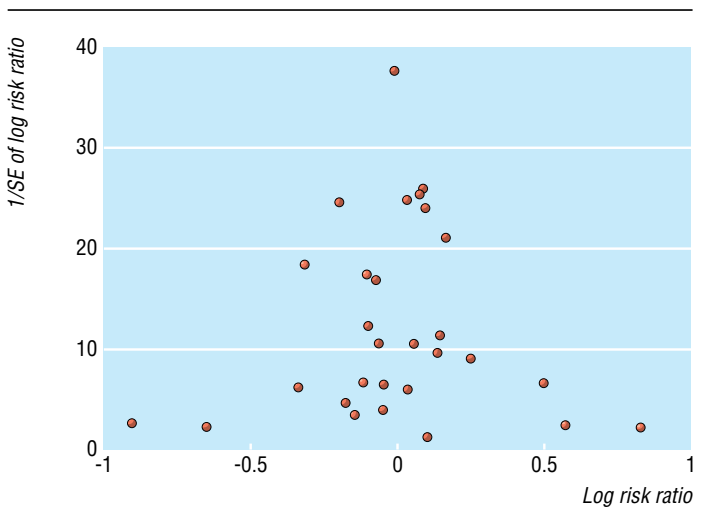

Fig 1 Funnel plot of extracted studies

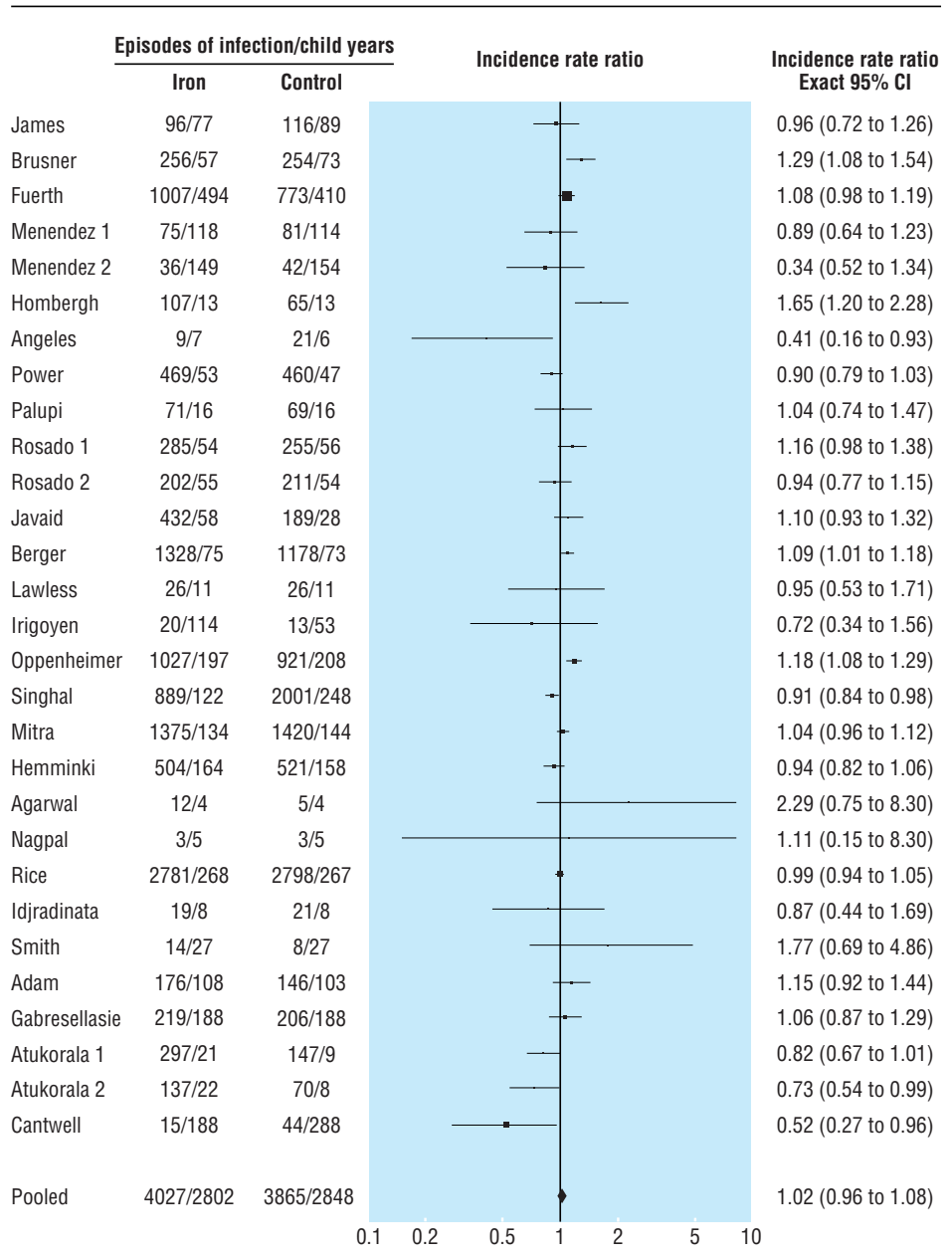

Heterogeneity $Q=78.29, d f=28, P<0.0001$

Fig 2 Forest plot for incidence rate ratio for all recorded illnesses

an equal distribution of fever in both groups would eliminate any bias and also prevent non-inclusion of any observed infection.

The methods of surveillance varied: 15 were clinic based whereas 13 were field trials with active surveillance for cases. If iron supplementation has selective effects on mild rather than more severe episodes of illness then differences in methods of case detection may influence the observed effects of iron supplementation.

\section{Bias detection for included studies}

The funnel plot (fig 1) seems symmetrical, and we found no evidence of bias using the Egger (weighted regression) method ( $\mathrm{P}=0.663$ for bias) or the Begg (rank correlation) method (continuity corrected $\mathrm{P}=0.488)$.

\section{Pooled and stratified estimates}

We collected data on 7892 children followed up for 5650 child years -4027 children and 2802 child years in the iron supplemented group and 3865 children and 2848 child years in the placebo group (table 3 ). The pooled estimate of the incidence rate ratio (iron versus placebo) for all the recorded morbidities was 1.02 (95\% confidence interval 0.96 to $1.08 ; \mathrm{P}=0.54$; test for heterogeneity $\mathrm{Q}=78.29, \mathrm{P}<0.0001$, fig 2). Calculations of incidence rate ratio based on "actual" data 
Table 2 Baseline characteristics of included trials (posted as supplied by author)

\begin{tabular}{|c|c|c|c|c|c|c|c|c|c|}
\hline & Location & $\begin{array}{l}\text { Age } \\
\text { group }\end{array}$ & $\begin{array}{l}\text { Sample } \\
\text { size } \\
\text { (total, } \\
\text { iron, } \\
\text { control) }\end{array}$ & $\begin{array}{l}\text { Method of } \\
\text { randomisation, } \\
\text { allocation } \\
\text { concealment, follow } \\
\text { up, blinding* }\end{array}$ & $\begin{array}{c}\text { Eligibility and exclusion } \\
\text { criteria }\end{array}$ & $\begin{array}{l}\text { Iron supplementation } \\
\text { (route, dose, duration of } \\
\text { supplementation, } \\
\text { duration of follow up, } \\
\text { intervention in } \\
\text { treatment group, control } \\
\text { group) }\end{array}$ & $\begin{array}{c}\text { Case } \\
\text { detection }\end{array}$ & Morbidities studied & $\begin{array}{c}\text { Case } \\
\text { definition }\end{array}$ \\
\hline James, $1960^{31}$ & USA & 1 month & $\begin{array}{l}\text { 181, } 84 \\
97\end{array}$ & Unclear, B, D, C & $\begin{array}{c}\text { Birth weight } \leqslant 2000 \mathrm{~g} \text {, } \\
\text { survival for more than } \\
24 \text { hours, weight }>2000 \\
\mathrm{~g}\end{array}$ & $\begin{array}{c}\text { Parenteral, } 50 \text { mg X 5, -, } \\
11 \text { months; T/t: Iron } \\
\text { dextran } \\
\text { C: No placebo }\end{array}$ & Clinic & $\begin{array}{l}\text { URTI, LRTI, } \\
\text { Diarrhoea }\end{array}$ & $\begin{array}{l}\text { Hospital } \\
\text { diagnosis }\end{array}$ \\
\hline Cantwell, $1972^{32}$ & New Zealand & 2 days & $\begin{array}{c}238,94 \\
144\end{array}$ & $\begin{array}{l}\text { By alternate days of } \\
\text { birth, D, A, C }\end{array}$ & $\begin{array}{l}\text { Maori babies (at least } \\
25 \% \text { Maori blood) } \\
\text { delivered at Hawkes Bay }\end{array}$ & $\begin{array}{c}\text { Parenteral, 50mg X 5, -, } \\
30 \text { months; T/t: Iron } \\
\text { dextran } \\
\text { C: No placebo }\end{array}$ & Clinic & $\begin{array}{l}\text { Pneumonia, URTI, } \\
\text { skin infections, } \\
\text { gastroenteritis }\end{array}$ & $\begin{array}{l}\text { Hospital } \\
\text { diagnosis }\end{array}$ \\
\hline Fuerth, $1974^{33}$ & USA & 1 month & $\begin{array}{l}602,329 \\
273\end{array}$ & $\begin{array}{l}\text { Alternate allocation, D, } \\
\text { D, A }\end{array}$ & $\begin{array}{c}\text { Full term } \\
\text { Exclusion: On iron } \\
\text { medication, vitamins, } \\
\text { received blood } \\
\text { transfusion, received less } \\
\text { than } 50 \% \text { supplements } \\
\text { between two visits, } \mathrm{Hb} \\
\text { dropped to }<80 \mathrm{~g} / \mathrm{l} \\
\text { during study }\end{array}$ & $\begin{array}{c}\text { Oral, } 30 \mathrm{mg} / \text { day, } 18 \\
\text { months, } 18 \text { months; T/t: } \\
\text { Ferrous sulphate } \\
\text { C: Placebo containing } \\
\text { bismuth }\end{array}$ & Clinic & Infectious illness & Not mentioned \\
\hline $\begin{array}{l}\text { Oppenheimer, } \\
1986^{34}\end{array}$ & $\begin{array}{l}\text { Papua New } \\
\text { Guinea }\end{array}$ & 2 months & $\begin{array}{l}486,236 \\
250\end{array}$ & $\begin{array}{l}\text { Matched pairs } \\
\text { randomised into } \\
\text { treatment and control } \\
\text { groups, B, C, B }\end{array}$ & Resident of Madang & $\begin{array}{l}\text { Parenteral, } 150 \mathrm{mg}, 10 \\
\text { mo } \\
\text { T/t: Iron dextran } \\
\text { C: Saline }\end{array}$ & $\begin{array}{l}\text { Field and } \\
\text { clinic }\end{array}$ & $\begin{array}{l}\text { URTI, LRTI, TB, } \\
\text { lung abscess, } \\
\text { malaria, } \\
\text { gastroenteritis, etc. }\end{array}$ & $\begin{array}{l}\text { RTI- WHO } \\
\text { classification } \\
\text { Malaria: } \\
\text { symptomatic } \\
\text { Rest: not } \\
\text { mentioned }\end{array}$ \\
\hline Harvey, $1989^{35}$ & $\begin{array}{l}\text { Papua New } \\
\text { Guinea }\end{array}$ & $\begin{array}{l}8-12 \\
\text { years }\end{array}$ & $\begin{array}{c}312,156 \\
156\end{array}$ & $\begin{array}{l}\text { Matched pairs } \\
\text { randomised into } \\
\text { treatment and control } \\
\text { groups, C, C, D }\end{array}$ & $\mathrm{Hb}=80-120 \mathrm{~g} / \mathrm{l}$ & $\begin{array}{c}\text { Oral, } 130 \mathrm{mg} / \text { day, } 4 \\
\text { months, } 6 \text { months; T/t: } \\
\text { Ferrous sulphate } \\
\text { C: Identical placebo } \\
\text { (75\% cellulose, } 25 \% \\
\text { lactose) }\end{array}$ & Field & Malaria prevalence & $\begin{array}{c}\text { PS for malaria } \\
+\end{array}$ \\
\hline Smith, $1989^{11}$ & Gambia & $\begin{array}{c}6 \\
\text { months-5 } \\
\text { years }\end{array}$ & $\begin{array}{c}213,106 \\
107\end{array}$ & Unclear, B, C, A & $\begin{array}{l}\mathrm{Hb}, \mathrm{MCV}<3 \text { rd centile of } \\
\text { reference population } \\
\text { Exclusion: } \mathrm{Hb}<50 \mathrm{~g} / \mathrm{l}\end{array}$ & $\begin{array}{c}\text { Oral, } 3-6 \mathrm{mg} / \mathrm{kg} / \text { day, } 3 \\
\text { months, } 3 \text { months } \\
\text { T/t: Ferrous sulphate in } \\
\text { orange juice } \\
\text { C: Orange juice }\end{array}$ & Field & Malaria & $\begin{array}{l}\text { Axillary temp } \\
>37.5^{\circ} \mathrm{C} \text { with } \\
\text { P. falciparum + }\end{array}$ \\
\hline Javaid, $1991^{37}$ & Pakistan & 4 months & $\begin{array}{c}129,87 \\
42\end{array}$ & Unclear, B, D, D & Birth weight $>2500 \mathrm{~g}$ & $\begin{array}{c}\text { Fortified, } 7.5 \mathrm{mg} / 100 \\
\text { mg, } 8 \text { months, } 8 \\
\text { months; T/t: Iron fortified } \\
\text { milk cereal } \\
\text { C: Milk cereal }\end{array}$ & Field & $\begin{array}{l}\text { URTI, LRTI, } \\
\text { diarrhoea }\end{array}$ & $\begin{array}{c}\text { LRTI: } \\
\text { significant } \\
\text { complaint with } \\
\text { +ve physical } \\
\text { examination; } \\
\text { Diarrhoea: }>4 \\
\text { loose } \\
\text { stools/day }\end{array}$ \\
\hline Irigoyen, $1991^{38}$ & USA & 6 months & $\begin{array}{l}334,228 \\
106\end{array}$ & Unclear, B, D, A & $\begin{array}{c}\mathrm{Hb} \leqslant 115 \mathrm{~g} / \mathrm{l} \\
\text { Exclusion: Prematurity, } \\
\text { milk allergy, failure to } \\
\text { thrive, } H I V+\text {, recent } H \\
\text { influenzae type b } \\
\text { meningitis, fed low iron } \\
\text { formula, exclusively } \\
\text { breastfed, primary } \\
\text { physician refusal }\end{array}$ & $\begin{array}{l}\text { Oral, } 3,6 \mathrm{mg} / \mathrm{kg} / \mathrm{day}, 3 \\
\text { months, } 3 \text { months; T/t: } \\
\text { Ferrous sulphate } \\
\text { C: Identical placebo } †\end{array}$ & Clinic & Diarrhoea & Not mentioned \\
\hline Chippaux, $1991^{39}$ & Togo & $\begin{array}{l}6-36 \\
\text { months }\end{array}$ & $\begin{array}{c}190,95 \\
95\end{array}$ & Unclear, B, D, A & $\mathrm{Hb} \geqslant 80 \mathrm{~g} / \mathrm{l}$ & $\begin{array}{l}\text { Oral, } 2.5 \mathrm{mg} / \mathrm{kg} / \mathrm{day}, 3 \\
\text { months, } 9 \text { months; T/t: } \\
\text { Iron Betainate } \\
\text { C: Identical placebo } \dagger\end{array}$ & Clinic & Malaria & Smear positive \\
\hline Brunser, $1993^{40}$ & Chile & 3 months & $\begin{array}{l}400,200 \\
200\end{array}$ & $\begin{array}{l}\text { Random numbers } \\
\text { table, } A, D, A\end{array}$ & $\begin{array}{l}\text { Birth weight } \geqslant 2500 \mathrm{~g}, \\
\mathrm{~W} / \mathrm{A} \geqslant 80 \% \text { or } 50 \text { th } \\
\text { centile, } \mathrm{Hb} \geqslant 105 \mathrm{~g} / \mathrm{l}\end{array}$ & $\begin{array}{l}\text { Fortified, } 12 \mathrm{mg} / \mathrm{l}, 6 \\
\text { months, } 6 \text { monthsl; T/t: } \\
\text { Iron enriched milk } \\
\text { C: Control milk }\end{array}$ & Field & Diarrhoea & $\begin{array}{c}>3 \text { liquid } \\
\text { stools/day or } \\
\text { maternal report }\end{array}$ \\
\hline Angeles, $1993^{41}$ & Indonesia & $2-5$ years & $\begin{array}{l}80,40 \\
40\end{array}$ & Unclear, B, B, A & $\begin{array}{c}\text { W/A z score between }-2 \\
\text { and }-3, \mathrm{Hb}=80-110 \mathrm{~g} / \mathrm{l} \text {, } \\
\text { ferritin }<120 \mu \mathrm{g} / \mathrm{l}\end{array}$ & $\begin{array}{c}\text { Oral, } 30 \mathrm{mg} / \text { day, } 2 \\
\text { months, } 2 \text { months; T/t: } \\
\text { Ferrous sulphate, } \\
\text { Vitamin C } \\
\text { C: Vitamin C }\end{array}$ & Field & $\begin{array}{l}\text { Fever, RTI, } \\
\text { Diarrhoea }\end{array}$ & $\begin{array}{c}\text { Fever: Temp } \\
>37^{\circ} \mathrm{C}, \\
\text { Diarrhoea: }>4 \\
\text { watery } \\
\text { stools/d, RTI: } \\
\text { not mentioned }\end{array}$ \\
\hline
\end{tabular}


Table 2 Baseline characteristics of included trials (posted as supplied by author) contd

\begin{tabular}{|c|c|c|c|c|c|c|c|c|c|}
\hline & Location & $\begin{array}{c}\text { Age } \\
\text { group }\end{array}$ & $\begin{array}{l}\text { Sample } \\
\text { size } \\
\text { (total, } \\
\text { iron, } \\
\text { control) }\end{array}$ & $\begin{array}{l}\text { Method of } \\
\text { randomisation, } \\
\text { allocation } \\
\text { concealment, follow } \\
\text { up, blinding* }\end{array}$ & $\begin{array}{c}\text { Eligibility and exclusion } \\
\text { criteria }\end{array}$ & $\begin{array}{l}\text { Iron supplementation } \\
\text { (route, dose, duration of } \\
\text { supplementation, } \\
\text { duration of follow up, } \\
\text { intervention in } \\
\text { treatment group, control } \\
\text { group) }\end{array}$ & $\begin{array}{c}\text { Case } \\
\text { detection }\end{array}$ & Morbidities studied & $\begin{array}{c}\text { Case } \\
\text { definition }\end{array}$ \\
\hline Lawless, $1994^{42}$ & Kenya & $\begin{array}{l}6-11 \\
\text { years }\end{array}$ & $\begin{array}{c}86,44 \\
42\end{array}$ & $\begin{array}{c}\text { Stratified } \\
\text { randomisation (by } \\
\text { gender and initial } \mathrm{Hb} \\
\text { value), C, A, A }\end{array}$ & $\begin{array}{c}\mathrm{Hb} \geqslant 80 \mathrm{~g} / \mathrm{l} \\
\text { Exclusion; Heavy } \\
\text { hookworm infection, } \\
\text { Blood in the urine } \\
\text { indicative of } S \\
\text { haematobium, dislike of } \\
\text { uji, absence at the time } \\
\text { of interval exams }\end{array}$ & $\begin{array}{c}\text { Oral, } 150 \text { mg/day, } 3 \\
\text { months, } 3 \text { months; T/t: } \\
\text { Ferrous sulphate } \\
\text { C: Identical placebo† }\end{array}$ & School & $\begin{array}{c}\text { Diarrhoea, cough, } \\
\text { malaria }\end{array}$ & $\begin{array}{l}\text { PS for MP+; } \\
\text { diarrhoea, } \\
\text { cough-not } \\
\text { mentioned }\end{array}$ \\
\hline $\begin{array}{l}\text { Idjradinata, } \\
1994^{10}\end{array}$ & Indonesia & $\begin{array}{c}12-18 \\
\text { months }\end{array}$ & $\begin{array}{l}47,24 \\
23\end{array}$ & $\begin{array}{l}\text { Random numbers } \\
\text { table, B, B, D }\end{array}$ & $\begin{array}{l}\text { Birth weight }>2.5 \mathrm{~kg} \text {, } \\
\text { singleton pregnancy, } \mathrm{Hb} \\
\geqslant 8 \mathrm{~g} / \mathrm{dL} \text {, wt, length and } \\
\text { head circumference } \\
\text { within } 2 \mathrm{SD} \text { of NCHS } \\
\text { standards. } \\
\text { Exc/usion: Congenital } \\
\text { malformation, major } \\
\text { perinatal complication, } \\
\text { jaundice treated with } \\
\text { phototherapy, hospital } \\
\text { admission, } \\
\text { supplementation with } \\
\text { micronutreints before } \\
\text { enrolment, chronic } \\
\text { illness, folic acid } \\
\text { deficiency, } \\
\text { haemoglobinopathy or } \\
\text { thalassaemia }\end{array}$ & $\begin{array}{c}\text { Oral, } 3 \text { mg/kg/day, } 4 \\
\text { months, } 4 \text { months; T/t: } \\
\text { Ferrous sulphate } \\
\text { C: Identical placebo† }\end{array}$ & Clinic & $\begin{array}{l}\text { URTI, LRTI, } \\
\text { gastroenteritis }\end{array}$ & $\begin{array}{l}\text { Paediatrician's } \\
\text { diagnosis }\end{array}$ \\
\hline Hemminki, $1995^{43}$ & Hungary & $<45$ days & $\begin{array}{l}322,164 \\
158\end{array}$ & Unclear, A, B, D & $\begin{array}{l}\text { Birth weight } \geqslant 2500 \mathrm{~g} \\
\text { Exclusion: Critically ill, } \\
\text { malformations, child } \\
\text { cared for outside home, } \\
\text { consultation with private } \\
\text { physician }\end{array}$ & $\begin{array}{l}\text { Fortified, } 6.5 \mathrm{mg} / \mathrm{l}, 10.5 \\
\text { months, } 10.5 \text { months; } \\
\text { T/t: Iron fortified formula } \\
\text { C: Non-fortified formula }\end{array}$ & Clinic & URTI, fever & Not mentioned \\
\hline $\begin{array}{l}\text { Van den } \\
\text { Hombergh, } \\
1996^{44}\end{array}$ & Tanzania & $\begin{array}{l}<30 \\
\text { months }\end{array}$ & $\begin{array}{l}100,50 \\
50\end{array}$ & Unclear, B, B, D & $\begin{array}{c}\mathrm{Hb} \leqslant 50 \mathrm{~g} / \mathrm{l}, \mathrm{PS} \text { for } \\
\mathrm{MP}+\text {. } \\
\text { Exclusion: Cerebral } \\
\text { malaria, Non-falciparum } \\
\text { malaria, sickle cell } \\
\text { anaemia, other } \\
\text { significant illness }\end{array}$ & $\begin{array}{l}\text { Oral, } 200 \mathrm{mg} / \text { day, } 3 \\
\text { months, } 3 \text { months; } \\
\text { T/t: Ferrous sulphate, } \\
\text { folic acid } \\
\text { C: Folic acid }\end{array}$ & Clinic & $\begin{array}{l}\text { Malaria, pneumonia, } \\
\text { other infections }\end{array}$ & $\begin{array}{l}\text { Malaria: smear } \\
\text { positive } \\
\text { Pneumonia, } \\
\text { other } \\
\text { infections: not } \\
\text { mentioned }\end{array}$ \\
\hline Adam, $1996^{45}$ & Ethiopia & $\begin{array}{c}6 \\
\text { months-7 } \\
\text { years }\end{array}$ & $\begin{array}{c}841,431 \\
410\end{array}$ & Unclear, B, B, A & $\mathrm{Hb}=60-110 \mathrm{~g} / \mathrm{l}$ & $\begin{array}{l}\text { Oral, } 3 \mathrm{mg} / \mathrm{kg} / \mathrm{day}, 3 \\
\text { months, } 3 \text { months; } \\
\text { T/t: Ferrous sulphate } \\
\text { C: Identical placebo† }\end{array}$ & Active & Malaria & Fever \\
\hline $\begin{array}{l}\text { Gebresellassie, } \\
1996^{46}\end{array}$ & Ethiopia & $\begin{array}{l}5-14 \\
\text { years }\end{array}$ & $\begin{array}{l}500,250 \\
250\end{array}$ & Unclear, B, C, A & $\begin{array}{l}\mathrm{Hb}=50-120 \mathrm{~g} / \mathrm{l} \\
P \text { falciparum -ve }\end{array}$ & $\begin{array}{c}\text { Oral, } 60 \mathrm{mg} / \text { day, } 3 \\
\text { months, } 6 \text { months } \\
\text { T/t: Ferrous sulphate } \\
\text { C: Identical placebo } †\end{array}$ & Active & Malaria & $\begin{array}{c}\text { Temp }>37.5^{\circ} \mathrm{C}, \\
\text { P. falciparum } \\
\text { +ve }\end{array}$ \\
\hline Mitra, $1997^{47}$ & Bangladesh & $\begin{array}{c}2-48 \\
\text { months }\end{array}$ & $\begin{array}{c}349,172 \\
177\end{array}$ & $\begin{array}{c}\text { Block randomisation of } \\
4 \text { homogeneous } \\
\text { clusters, } A, C, A\end{array}$ & $\begin{array}{l}\text { Exclusion: Critically ill, } \\
\text { congenital } \\
\text { malformations, metabolic } \\
\text { disorders }\end{array}$ & $\begin{array}{c}\text { Oral, } 15 \mathrm{mg} / \text { day, } 15 \\
\text { months, } 15 \text { months } \\
\text { T/t: Ferrous gluconate, } \\
\text { vitamins } \ddagger \\
\text { C: Vitamins } \ddagger\end{array}$ & Field & $\begin{array}{c}\text { Diarrhoea, } \\
\text { dysentery, ARI }\end{array}$ & $\begin{array}{c}\text { Diarrhoea: >2 } \\
\text { liquid stools/d } \\
\text { and maternal } \\
\text { report; } \\
\text { Dysentery: } \\
\text { blood in } \\
\text { stools; ARI: } \\
>50 \mathrm{bpm} \text { in } \\
\text { child <1 yr, } \\
>40 \mathrm{bpm} \text { in } \\
\text { child } 12-15 \\
\text { months }\end{array}$ \\
\hline Palupi, $1997^{48}$ & Indonesia & $2-5$ years & $\begin{array}{l}194,96 \\
98\end{array}$ & Unclear, B, B, A & $\begin{array}{l}\text { Registered at village } \\
\text { health centre }\end{array}$ & $\begin{array}{l}\text { Oral, } 15 \mathrm{mg} / \text { week, } 2 \\
\text { months, } 2 \text { months } \\
\text { T/t: Ferrous sulphate } \\
\text { C: Identical placebo† }\end{array}$ & Clinic & Worm infestation & $\begin{array}{c}\text { Stool } \\
\text { microscopy + }\end{array}$ \\
\hline Rosado, $1997^{49}$ & Mexico & $\begin{array}{l}1.5-3 \\
\text { years }\end{array}$ & $\begin{array}{l}219,109 \\
110\end{array}$ & $\begin{array}{c}\text { Stratified } \\
\text { randomisation (by age } \\
\text { and sex), B, C, A }\end{array}$ & Age as stated & $\begin{array}{c}\text { Oral, } 20 \mathrm{mg} / \text { day, } 12 \\
\text { months, } 12 \text { months } \\
\text { Group } 1 \\
\text { T/t: Ferrous sulphate } \\
\text { C: Placebo } † \\
\text { Group } 2 \\
\text { T/t: Ferrous sulphate, } \\
\text { zinc methionine } \\
\text { C: Zinc methionine }\end{array}$ & Field & RTI, diarrhoea, fever & $\begin{array}{c}\text { RTI: runny } \\
\text { nose, common } \\
\text { cold, sore } \\
\text { throat, cough; } \\
\text { Diarrhoea, } \\
\text { Fever: maternal } \\
\text { reporting }\end{array}$ \\
\hline
\end{tabular}


Table 2 Baseline characteristics of included trials (posted as supplied by author) contd

\begin{tabular}{|c|c|c|c|c|c|c|c|c|c|}
\hline & Location & $\begin{array}{c}\text { Age } \\
\text { group }\end{array}$ & $\begin{array}{c}\text { Sample } \\
\text { size } \\
\text { (total, } \\
\text { iron, } \\
\text { control) } \\
\end{array}$ & $\begin{array}{l}\text { Method of } \\
\text { randomisation, } \\
\text { allocation } \\
\text { concealment, follow } \\
\text { up, blinding }\end{array}$ & $\begin{array}{c}\text { Eligibility and exclusion } \\
\text { criteria }\end{array}$ & $\begin{array}{l}\text { Iron supplementation } \\
\text { (route, dose, duration of } \\
\text { supplementation, } \\
\text { duration of follow up, } \\
\text { intervention in } \\
\text { treatment group, control } \\
\text { group) }\end{array}$ & $\begin{array}{c}\text { Case } \\
\text { detection }\end{array}$ & Morbidities studied & $\begin{array}{c}\text { Case } \\
\text { definition }\end{array}$ \\
\hline Menendez, $1997^{50}$ & Tanzania & 2 months & $\begin{array}{c}832,417 \\
415\end{array}$ & $\begin{array}{c}\text { Block randomisation, } \\
\text { A, D, A }\end{array}$ & $\begin{array}{l}\text { Birth weight }>1500 \mathrm{~g} \text {, } \\
\text { PCV }>25 \% \text { at } 8 \text { weeks. } \\
\text { Exclusion: Congenital } \\
\text { malformation, congenital } \\
\text { or neonatal infection. }\end{array}$ & $\begin{array}{c}\text { Oral, } 2 \mathrm{mg} / \mathrm{kg} / \mathrm{day}, 4 \\
\text { months, } 10 \text { months } \\
\text { Group } 1 \\
\text { T/t: Ferrous glycine } \\
\text { sulphate, placebo syrup } \ddagger \\
\text { C: Placebo syrups } \ddagger \\
\text { Group } 2 \\
\text { T/t: Iron syrup, } \\
\text { Deltaprim } \\
\text { C: Deltaprim, placebo } \\
\text { syrup } \ddagger\end{array}$ & Clinic & Malaria & $\begin{array}{c}\text { Axillary temp } \\
>37.5^{\circ} \mathrm{C} \text { with } P \\
\text { falciparum +ve }\end{array}$ \\
\hline $\begin{array}{l}\text { Rice, } 1999 \\
\quad \text { (unpublished) }\end{array}$ & Tanzania & $\begin{array}{l}\text { 3-56 } \\
\text { months }\end{array}$ & $\begin{array}{c}614,307 \\
307\end{array}$ & $\begin{array}{l}\text { Randomisation of } \\
\text { households of the } \\
\text { study area into two } \\
\text { groups, B, A, B }\end{array}$ & Age as stated & $\begin{array}{c}\text { Oral, } 10 \mathrm{mg} / \text { day, } 12 \\
\text { months, } 12 \text { months } \\
\text { T/t: Iron sulphate } \\
\text { C: Identical placebo† }\end{array}$ & Field & $\begin{array}{l}\text { Diarrhoea, } \\
\text { dysentery, RTI, } \\
\text { malaria, fever }\end{array}$ & $\begin{array}{c}\text { RTI: cough } \\
\text { with difficult } \\
\text { breathing; } \\
\text { Diarrhoea: }>3 \\
\text { liquid stools/ } \\
\text { day; Dysentery: } \\
\text { blood in stools }\end{array}$ \\
\hline $\begin{array}{l}\text { Agarwal, } 1999 \\
\text { (unpublished) }\end{array}$ & India & $\begin{array}{c}50-80 \\
\text { days }\end{array}$ & $\begin{array}{c}73,37 \\
36\end{array}$ & $\begin{array}{c}\text { Computer generated } \\
\text { random numbers, A, } \\
\text { C, A }\end{array}$ & $\begin{array}{l}\text { Gestation } \geqslant 37 \text { weeks, } \\
\text { birth weight }<2500 \mathrm{~g} \text {. } \\
\text { Exclusion: Twins, } \\
\text { congenital } \\
\text { malformations, received } \\
\text { blood, adverse neonatal } \\
\text { event requiring } \\
\text { admission in nursery, } \\
\text { sampling before } \\
\text { recruitment }>10 \mathrm{ml} \text {, } \\
\text { significant current } \\
\text { morbidity, maternal APH }\end{array}$ & $\begin{array}{c}\text { Oral, } 3 \mathrm{mg} / \mathrm{kg} / \text { day, } 2 \\
\text { months, } 2 \text { months } \\
\text { T/t: Ferric ammonium } \\
\text { citrate } \\
\text { C: Identical placebo }\end{array}$ & Clinic & RTI & $\begin{array}{c}\text { Maternal report } \\
\text { as interpreted } \\
\text { by } \\
\text { paediatrician }\end{array}$ \\
\hline $\begin{array}{l}\text { Nagpal, } 2000 \\
\quad \text { (unpublished) }\end{array}$ & India & $\begin{array}{c}4-6 \\
\text { months }\end{array}$ & $\begin{array}{c}100,49 \\
51\end{array}$ & $\begin{array}{c}\text { Computer generated } \\
\text { random numbers, A, } \\
\text { D, A }\end{array}$ & $\begin{array}{l}\text { Gestation } \geqslant 37 \text { weeks, } \\
\text { birth weight } \geqslant 2500 \mathrm{~g} \text {, } \\
\text { breast fed } \\
\text { Exclusion: Twins, } \\
\text { congenital } \\
\text { malformations, received } \\
\text { blood or iron, adverse } \\
\text { neonatal event requiring } \\
\text { admission in nursery, } \\
\text { sampling before } \\
\text { recruitment }>10 \mathrm{ml}, \\
\text { significant current } \\
\text { morbidity }\end{array}$ & $\begin{array}{c}\text { Oral, } 2.5 \mathrm{mg} / \mathrm{kg} / \mathrm{day}, 2 \\
\text { months, } 2 \text { months } \\
\text { T/t: Ferric ammonium } \\
\text { citrate } \\
\text { C: Identical placebo }\end{array}$ & Clinic & $\begin{array}{l}\text { RTI, diarrhoea, } \\
\text { others }\end{array}$ & $\begin{array}{c}\text { Maternal report } \\
\text { as interpreted } \\
\text { by } \\
\text { paediatrician }\end{array}$ \\
\hline Berger, $2000^{51}$ & Togo & $\begin{array}{c}\text { 6-36 } \\
\text { months }\end{array}$ & $\begin{array}{l}\text { 197, } 100 \\
\quad 97\end{array}$ & Unclear, B, C, B & $\mathrm{Hb} \geqslant 80 \mathrm{~g} / \mathrm{l}$ & $\begin{array}{c}\text { Oral, 2-3mg/kg/day, } 3 \\
\text { months, } 9 \text { months } \\
\text { T/t: Iron Betainate } \\
\text { C: Identical placebo† }\end{array}$ & Field & $\begin{array}{l}\text { URTI, LRTI, malaria, } \\
\text { diarrhoea, } \\
\text { cutaneous infection, } \\
\text { fever, worms }\end{array}$ & Not mentioned \\
\hline Singhal, $2000^{52}$ & UK & 9 months & $\begin{array}{c}493,162 \\
331\end{array}$ & $\begin{array}{c}\text { Separate } \\
\text { randomisation for } \\
\text { Asians and } \\
\text { non-Asians, A, C, A }\end{array}$ & $\begin{array}{l}\text { Birth weight }>2500 \mathrm{~g} \text {, } \\
\text { gestation }>36 \text { weeks. } \\
\text { Exclusion: Severe } \\
\text { chronic disease, } \\
\text { congenital anomalies, } \\
\text { haematologic disorders, } \\
\text { previously received iron } \\
\text { or blood }\end{array}$ & $\begin{array}{l}\text { Fortified, } 12 \mathrm{mg} / \mathrm{L}, 9 \\
\text { months, } 9 \text { months; } \\
\text { T/t: Iron fortified formula } \\
\text { C: Cows' milk or } \\
\text { standard formula }\end{array}$ & Clinic & $\begin{array}{l}\text { Chest infection, } \\
\text { URTI, others }\end{array}$ & $\begin{array}{l}\text { URTI, } \\
\text { diarrhoea: } \\
\text { maternal } \\
\text { report; chest } \\
\text { infection: } \\
\text { treatment with } \\
\text { antibiotics }\end{array}$ \\
\hline $\begin{array}{r}\text { Atukorala, } 2001 \\
\text { (unpublished) }\end{array}$ & Sri Lanka & $\begin{array}{c}5-10 \\
\text { years }\end{array}$ & $\begin{array}{c}364,262 \\
102\end{array}$ & Unclear, B, C, A & $\begin{array}{l}\text { Outpatients at children's } \\
\text { hospital }\end{array}$ & $\begin{array}{c}\text { Oral, } 60 \mathrm{mg} / \text { day, } 2 \\
\text { months, } 2 \text { months } \\
\text { T/t: Ferrous sulphate } \\
\text { C: Lactose }\end{array}$ & Field & URTI, diarrhoea & $\begin{array}{c}\text { URTI: clinical } \\
\text { evidence with } \\
\text { inflammatory } \\
\text { parameters; } \\
\text { diarrhoea: >2 } \\
\text { semisolid } \\
\text { watery } \\
\text { stools/day }\end{array}$ \\
\hline
\end{tabular}

$\mathrm{ARI}=$ acute respiratory illness; bpm=breaths per minute; $\mathrm{C}=$ =intervention in the control group; GI=gastrointestinal; $\mathrm{Hb}=$ haemoglobin, LRTI=lower respiratory tract infection; $\mathrm{MCV}=$ mean corpuscular volume; MP=malarial parasite; $P$ falciparum=Plasmodium falciparum; PS=peripheral smear; RTl=respiratory tract infection; TB=tuberculosis; URTl=upper respiratory tract infection;

$T / t=$ intervention in the treatment group;

${ }^{*}$ Allocation concealment: (A) adequate; (B) unclear; (C) inadequate; (D) not used. Completeness of follow up: (A) <3\% of participants excluded; (B) $3 \%$ to $9.9 \%$ of participants excluded; (C)

$10 \%$ to $19.9 \%$ of participants excluded; (D) $20 \%$ or more of participants excluded. Blinding: (A) double blinding; (B) single blinding; (C) no blinding; (D) unclear.

†Mentioned by the authors as being identical in appearance and/or taste; exact composition not mentioned.

‡Exact composition not mentioned.

(when available) and computations from sample size at the end of the study $(1.03,0.97$ to $1.08, \mathrm{P}=0.21$; test for heterogeneity $\mathrm{Q}=72.19, \quad \mathrm{P}<0.0001)$ were virtually identical with computations based on sample sizes at the beginning of the study. Besides the incidence rate ratio, from the public health perspective the incidence rate difference is considered to be more informative. The incidence rate difference (iron minus placebo) for all the recorded illnesses was 0.06 episodes per child 
Table 3 Extracted data from included studies. Episodes of infection and exposure time (in child years) (posted as supplied by author)

\begin{tabular}{|c|c|c|c|c|c|c|c|c|c|c|c|c|c|c|c|c|c|c|c|c|}
\hline \multirow[b]{3}{*}{ Study } & \multicolumn{4}{|c|}{ Total infections } & \multicolumn{4}{|c|}{ Diarrhoea } & \multicolumn{4}{|c|}{ Respiratory tract infection } & \multicolumn{4}{|c|}{ Malaria } & \multicolumn{4}{|c|}{ Other infections } \\
\hline & \multicolumn{2}{|c|}{ Iron } & \multicolumn{2}{|c|}{ Control } & \multicolumn{2}{|c|}{ Iron } & \multicolumn{2}{|c|}{ Control } & \multicolumn{2}{|c|}{ Iron } & \multicolumn{2}{|c|}{ Control } & \multicolumn{2}{|c|}{ Iron } & \multicolumn{2}{|c|}{ Control } & \multicolumn{2}{|r|}{ Iron } & \multicolumn{2}{|c|}{ Control } \\
\hline & Epi & Obs & Epi & Obs & Epi & Obs & Epi & Obs & Epi & Obs & Epi & Obs & Epi & Obs & Epi & Obs & Epi & Obs & Epi & Obs \\
\hline James $^{31}$ & 96 & 77 & 116 & 88.91 & 16 & 77 & 25 & 88.91 & 80 & 77 & 91 & 88.91 & & & & & & & & \\
\hline Fuerth $^{33}$ & 1007 & 493.5 & 773 & 409.5 & & & & & & & & & & & & & 1007 & 493.5 & 773 & 409.5 \\
\hline Oppenheimer $^{34}$ & 1027 & 196.66 & 921 & 208.33 & 44 & 196.66 & 37 & 208.33 & 498 & 196.66 & 435 & 208.33 & 82 & 196.66 & 66 & 208.33 & 403 & 196.66 & 383 & 208.33 \\
\hline Smith $^{11}$ & 14 & 26.5 & 8 & 26.75 & & & & & & & & & 14 & 26.5 & 8 & 26.75 & & & & \\
\hline Power $^{36}$ & 469 & 52.5 & 460 & 46.5 & 84 & 52.5 & 91 & 46.5 & 105 & 52.5 & 88 & 46.5 & & & & & 280 & 52.5 & 281 & 46.5 \\
\hline Javaid $^{37}$ & 432 & 58 & 189 & 28 & 250 & 58 & 105 & 28 & 182 & 58 & 84 & 28 & & & & & & & & \\
\hline |rigoyen $^{38}$ & 20 & 114 & 13 & 53 & 20 & 114 & 13 & 53 & & & & & & & & & & & & \\
\hline Brunser ${ }^{40}$ & 256 & 56.88 & 254 & 72.57 & 256 & 56.88 & 254 & 72.57 & & & & & & & & & & & & \\
\hline Angeles $^{41}$ & 9 & 6.5 & 21 & 6.16 & 2 & 6.5 & 6 & 6.16 & 4 & 6.5 & 10 & 6.16 & & & & & 3 & 6.5 & 5 & 6.16 \\
\hline Lawless $^{42}$ & 26 & 11 & 26 & 10.5 & 7 & 11 & 8 & 10.5 & 19 & 11 & 18 & 10.5 & & & & & & & & \\
\hline Idjradinata $^{10}$ & 19 & 8 & 21 & 7.66 & & & & & & & & & & & & & 19 & 8 & 21 & 7.66 \\
\hline Hemminki ${ }^{43}$ & 504 & 164 & 521 & 158 & & & & & 288 & 164 & 305 & 158 & & & & & 216 & 164 & 216 & 158 \\
\hline $\begin{array}{l}\text { Van den } \\
\text { Hombergh }{ }^{44}\end{array}$ & 107 & 12.5 & 65 & 12.5 & & & & & 26 & 12.5 & 5 & 12.5 & & & & & 81 & 12.5 & 60 & 12.5 \\
\hline Gabresellasie $^{46}$ & 219 & 187.5 & 206 & 187.5 & & & & & & & & & 219 & 187.5 & 206 & 187.5 & & & & \\
\hline Mitra $^{47}$ & 1375 & 134 & 1420 & 143.5 & 670 & 127 & 695 & 139 & 705 & 141 & 725 & 148 & & & & & & & & \\
\hline Palupi 48 & 71 & 15.5 & 69 & 15.66 & & & & & & & & & & & & & 71 & 15.5 & 69 & 15.66 \\
\hline Rosado $1^{49}$ & 285 & 54 & 255 & 56 & 76 & 54 & 62 & 56 & 192 & 54 & 179 & 56 & & & & & 17 & 54 & 14 & 56 \\
\hline Rosado $2^{49}$ & 202 & 55 & 211 & 54 & 46 & 55 & 40 & 54 & 139 & 55 & 163 & 54 & & & & & 17 & 55 & 8 & 54 \\
\hline Menendez $1^{50}$ & 75 & 118.4 & 81 & 113.8 & & & & & & & & & 75 & 118.4 & 81 & 113.8 & & & & \\
\hline Menendez $2^{50}$ & 36 & 148.5 & 42 & 145.4 & & & & & & & & & 36 & 148.5 & 42 & 145.4 & & & & \\
\hline $\begin{array}{l}\text { Rice } \\
\text { (unpublished) }\end{array}$ & 2781 & 267.97 & 2798 & 267.39 & 388 & 267.98 & 376 & 267.37 & 1006 & 267.98 & 995 & 267.37 & & & & & 1387 & 267.97 & 1427 & 267.39 \\
\hline $\begin{array}{l}\text { Agarwal } \\
\text { (unpublished) }\end{array}$ & 12 & 3.75 & 5 & 3.58 & & & & & 12 & 3.75 & 5 & 3.58 & & & & & & & & \\
\hline $\begin{array}{l}\text { Nagpal } \\
\text { (unpublished) }\end{array}$ & 3 & 4.5 & 3 & 5 & 2 & 4.5 & 2 & 5 & 1 & 4.5 & 0 & 5 & & & & & 0 & 4.5 & 1 & 5 \\
\hline Berger $^{51}$ & 1328 & 75 & 1178 & 72.75 & 211 & 75 & 127 & 72.75 & 623 & 75 & 627 & 72.75 & & & & & 494 & 75 & 424 & 72.75 \\
\hline Singhal ${ }^{52}$ & 889 & 121.5 & 2001 & 248.25 & 66 & 121.5 & 132 & 248.25 & 823 & 121.5 & 1869 & 248.25 & & & & & & & & \\
\hline $\begin{array}{l}\text { Atukorala } 1 \\
\text { (unpublished) }\end{array}$ & 297 & 21.33 & 147 & 8.66 & 23 & 21.33 & 5 & 8.66 & 274 & 21.33 & 142 & 8.66 & & & & & & & & \\
\hline $\begin{array}{l}\text { Atukorala } 2 \\
\quad \text { (unpublished) }\end{array}$ & 137 & 22.33 & 70 & 8.33 & 8 & 22.33 & 2 & 8.33 & 129 & 22.33 & 68 & 8.33 & & & & & & & & \\
\hline
\end{tabular}

Epi=no of episodes of infections observed. Obs=0bservation/exposure time in child years. RTl=respiratory tract infections.

year $(-0.06$ to $0.18, \mathrm{P}=0.34$; test for heterogeneity $\mathrm{Q}=$ 80.01, $\mathrm{P}<0.0001)$.

Stratified analysis for the effect on individual infectious illnesses showed that children in the iron supplementation group had an $11 \%(1 \%$ to $23 \%)$ higher risk (incidence rate ratio) of developing diarrhoea $(\mathrm{P}=0.04$; test for heterogeneity $\mathrm{Q}=30.24, \mathrm{P}=0.04$, table 4$)$. The effect on other individual illnesses was not significant. However, the incidence rate difference (public health impact) for diarrhoea was 0.05 episodes per child year $(-0.03$ to $0.13, \mathrm{P}=0.21$; test for heterogeneity $\mathrm{Q}=42.03$, $\mathrm{P}=0.001)$. Further stratification showed that the significantly increased risk of diarrhoea associated with iron supplementation was restricted to oral supplementation (nine studies; incidence rate ratio $1.15,1.01$ to 1.32, $\mathrm{P}=0.04$; incidence rate difference 0.18 episodes per child year, -0.01 to $0.37 ; \mathrm{P}=0.07$ ). The individual studies had not determined the cause of the diarrhoea, though dysentery indicates severe infectious diarrhoea. Only two studies provided information on dysentery; they showed no difference in the incidence between the two groups. Meta-regression showed that the route of iron administration (oral versus other) was not significantly associated with incidence rate ratio for diarrhoea (risk ratio $1.06,0.85$ to $1.32, \mathrm{P}=0.59$ ).

From the available data we found no increased risk of severe illness associated with iron supplementation (analysis possible only for lower respiratory tract infection and dysentery).

\section{Malarial parasitaemia}

Table 5 shows the data extracted on malarial parasitaemia. The pooled odds ratio for positive smear tests for malaria at the end of the supplementation period (random effects model) was 1.43 (1.08 to $1.91, \mathrm{P}=0.014$; test for heterogeneity $\mathrm{Q}=11.611, \mathrm{P}=0.114$, fig 3. Metaregression analysis of trials with relevant data (excluding the study by Oppenheimer et $\mathrm{al}^{34}$ ) indicated that this treatment effect was significantly associated with the baseline positivity of smear tests (for a unit increase in log odds ratio of baseline positivity, the treatment effect increased by $2.89 ; 1.37$ to $6.10 ; \mathrm{P}=0.005)$ but not iron supplementation $(1.24 ; 0.98$ to $1.57 ; \mathrm{P}=0.076)$.

Table 4 Pooled estimates (incidence rate ratio) of effect of iron supplementation on total and individual infections

\begin{tabular}{|c|c|c|c|c|}
\hline Infection type & No of trials & $\begin{array}{l}\text { Random effects model } \\
\qquad(95 \% \mathrm{Cl})\end{array}$ & $P$ value & $\begin{array}{l}\text { Tests for heterogeneity } \\
\text { (P value) }\end{array}$ \\
\hline Diarrhoea & 17 & 1.11 (1.01 to 1.23$)$ & 0.04 & $30.24(0.04)$ \\
\hline Non-diarrhoeal & 24 & $0.97(0.95$ to 1.06$)$ & 0.99 & $63.05(<0.0001)$ \\
\hline Respiratory tract & 17 & 0.98 (0.90 to 1.06$)$ & 0.54 & $53.18(<0.0001)$ \\
\hline Malaria & 5 & 1.07 (0.94 to 1.24$)$ & 0.35 & $5.58(0.35)$ \\
\hline Other infections ${ }^{*}$ & 13 & 1.04 (0.98 to 1.11$)$ & 0.20 & $18.15(0.15)$ \\
\hline Lower respiratory tract $†$ & 8 & 0.97 (0.83 to 1.23$)$ & 0.93 & $21.91(0.003)$ \\
\hline Dysentery† & 2 & 1.00 (0.87 to 1.15$)$ & 0.99 & $0.02(0.90)$ \\
\hline
\end{tabular}


Table 5 Extracted data from trials depicting prevalence of smears positive for malaria

\begin{tabular}{|c|c|c|c|c|}
\hline \multirow[b]{2}{*}{ Study } & \multicolumn{2}{|c|}{ Baseline } & \multicolumn{2}{|c|}{ End } \\
\hline & Iron & Control & Iron & Control \\
\hline Hombergh $^{44}$ & $50 / 50$ & $50 / 50$ & $13 / 47$ & $13 / 47$ \\
\hline Berger et a ${ }^{51}$ & $59 / 100$ & $62 / 97$ & $40 / 84$ & $42 / 79$ \\
\hline Rice (unpublished) & $258 / 316$ & $253 / 295$ & 233/279 & $221 / 256$ \\
\hline Chippaux $^{39}$ & $72 / 120$ & $74 / 120$ & $59 / 120$ & $66 / 120$ \\
\hline Smith $^{11}$ & $23 / 106$ & $19 / 107$ & $28 / 97$ & $16 / 90$ \\
\hline Harvey $^{35}$ & $119 / 159$ & $103 / 159$ & 78/141 & $57 / 138$ \\
\hline Gebresellassie $^{46}$ & $30 / 239$ & $31 / 241$ & $23 / 239$ & $17 / 241$ \\
\hline Oppenheimer ${ }^{34}$ & - & - & $37 / 200$ & $24 / 212$ \\
\hline
\end{tabular}

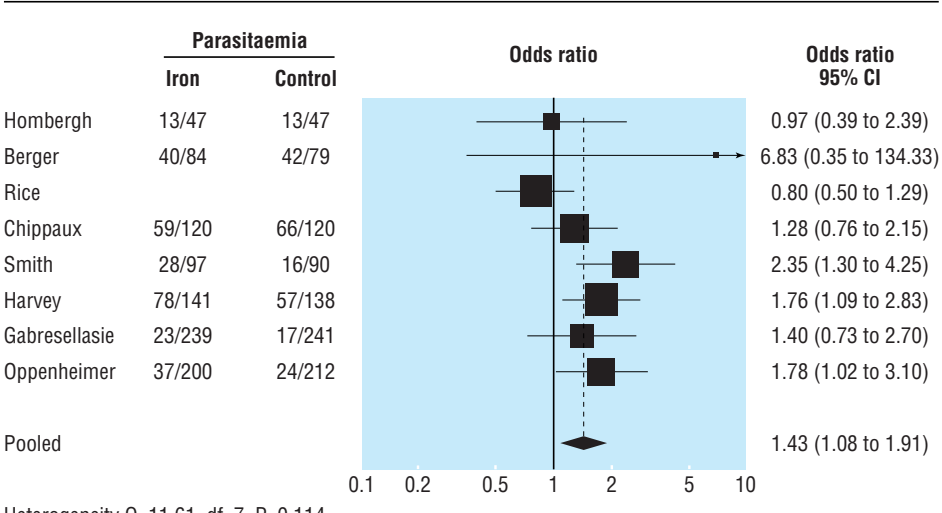

Heterogeneity $\mathrm{Q}=11.61, \mathrm{df}=7, \mathrm{P}=0.114$

Fig 3 Forest plot for odds ratio of malarial parasitaemia (positive results on blood smear test) at end of supplementation period

Meta-regression analyses to explore heterogeneity Stratified estimates indicated that iron supplementation did not significantly $(\mathrm{P}>0.05)$ increase the incidence of infections (incidence rate ratio and incidence rate difference), irrespective of the quality of methods, methods of surveillance, route of iron supplementation, duration of supplementation, geographic location of the study population, or the basal haemoglobin concentration of the iron supplemented group (data not presented). Meta-regression analysis showed that the treatment effect (incidence rate ratio) was not significantly associated with any of these study characteristics (table 6).

\section{Discussion}

The results from our analysis of these studies show that iron supplementation does not significantly increase the risk of overall infection. However, there was an increase in the risk of developing diarrhoea, but this would not have an important overall impact on public health. The occurrence of other illnesses and malarial parasitaemia (adjusted for positive smear results at baseline) was not significantly affected by iron administration $(\mathrm{P}>0.05)$.

\section{Strengths and limitations of analysis}

Despite wide clinical and methodological heterogeneity in the various trials, the main inference remained stable for the various sensitivity analyses that we performed. An important caveat is the lack of uniform definitions for the individual clinical morbidities. Uniform definitions and active surveillance would have provided greater weight to the conclusions. Furthermore, not all the included trials were of high quality. We could not explain the statistical heterogeneity by various study characteristics.

There are still some questions unanswered and some new issues raised. We could not determine whether the higher risk of diarrhoea was a result of increased gastrointestinal infections or a consequence of the irritant effect of iron on the gut motility, a known effect. ${ }^{53}$ Dysentery is invariably infective in origin, and the two trials that provided information found no evidence of an increase in dysentery in children receiving iron supplements.

We could not analyse the effect of dose on the incidence of infections. However, the near absence of any important adverse effects, particularly diarrhoea, in children receiving fortified foods (compared with medicinal iron) raises the possibility of a dose related effect. Interestingly, there was also a similar significant protective effect against the development of respiratory tract infections (four studies; incidence rate ratio $=0.92 ; 0.86$ to $0.98 ; \mathrm{P}=0.02$ ). However, our meta-regression analysis showed that the route of administration was not significantly associated with incidence rate ratio. Fortification with low doses of iron is closest to the physiological situation and could theoretically be considered the safest public health intervention. There is thus a case for concomitant evaluation of the possible beneficial effects of iron fortified foods on the haematological response and infections.

Meta-regression analysis suggested that the risk of acquiring infectious illnesses is inversely associated with the baseline haemoglobin concentration. Stratified analysis also suggested increased risk of infections in children who had a mean baseline concentration below $100 \mathrm{~g} / \mathrm{l}$. Iron supplementation promotes production of free radicals, and this may have a deleterious effect on the immunity of a child. Ironically, defences against free radicals are compromised the

Table 6 Meta-regression analyses for incidence rate ratio (IRR)

\begin{tabular}{|c|c|c|}
\hline Characteristic & $\begin{array}{l}\text { Univariate analysis IRR }(95 \% \mathrm{CI}) \text {, } \\
\text { P value }\end{array}$ & $\begin{array}{l}\text { Controlled for all variables IRR } \\
(95 \% \mathrm{CI}), \mathrm{P} \text { value }\end{array}$ \\
\hline \multicolumn{3}{|l|}{ Quality of study: } \\
\hline Allocation concealment (not adequate $v$ adequate) & $1.02(0.90$ to 1.16$), 0.716$ & 0.94 (0.72 to 1.22$), 0.624$ \\
\hline Completeness of follow up ( $\geqslant 10 \%$ participants excluded $v<10 \%$ excluded) & 1.01 (0.89 to 1.16$), 0.839$ & $1.17(0.92$ to 1.50$), 0.202$ \\
\hline Blinding (not double blind $v$ double blind) & 1.06 (0.95 to 1.19$), 0.298$ & $1.11(0.91$ to 1.36$), 0.287$ \\
\hline Morbidity surveillance (passive $v$ active) & 0.94 (0.84 to 1.05$), 0.266$ & 1.03 (0.83 to 1.27$), 0.809$ \\
\hline Route of supplementation (oral or parenteral $v$ fortified) & 1.04 (0.92 to 1.17$), 0.555$ & $1.03(0.82$ to 1.30$), 0.815$ \\
\hline Geographic location (developed ${ }^{*}$ v Asian or African countries) & 0.98 (0.87 to 1.11), 0.759 & 1.05 (0.81 to 1.36$), 0.723$ \\
\hline Unit increase in baseline haemoglobin status of iron supplemented group $(\mathrm{g} / \mathrm{l})$ & 0.97 (0.94 to 1.01$), 0.151$ & 0.95 (0.90 to 1.00$), 0.059$ \\
\hline Unit increase in duration of supplementation (months) & 1.00 (0.99 to 1.02$), 0.864$ & 0.99 (0.97 to 1.03$), 0.921$ \\
\hline
\end{tabular}

"Europe, North America, South America, and Australia and New Zealand. 


\section{What is already known on this topic}

Iron supplementation is recommended to prevent iron deficiency, which is a major health problem, especially in the developing countries

Conflicting data exist regarding the possibility of an increase in the incidence of infections with iron supplementation, resulting in concern about the safety of this intervention

\section{What this study adds}

Iron supplementation has no apparent harmful effect on the overall incidence of infectious illnesses in children

Iron administration increases the risk of developing diarrhoea

Fortification of foods may be the safest and most beneficial mode of supplementation in relation to infectious illnesses

most in iron deficiency and malnutrition, ${ }^{54}{ }^{55}$ which are conditions likely to benefit the most from iron supplementation. Interestingly, all the studies included in this stratified subset were from regions of the African continent where malaria is endemic. Some data suggest indirectly that iron deficiency in such regions decreases the susceptibility to disease related to malaria, HIV, and tuberculosis. ${ }^{56}$ The safety of iron supplementation in people with anaemia, particularly in regions where malaria is endemic, may be difficult to determine because of the ethical problem of withholding treatment in a control group.

We thank Sunil Sinha for facilitating access to Embase and two $\mathrm{PhD}$ dissertations. We also thank L Satyanaryana for help in conducting the meta-regression analysis. Part of this paper was presented as a poster at the International Nutritional Anaemia Consultative Group symposium 2001, held at Hanoi, Vietnam.

Contributors: TG prepared the protocol, applied the search strategy, performed the retrieval of articles, and extracted the data from the included studies. HPSS developed the idea for the review, finalised the protocol and search strategy, and performed the statistical analysis. Both the authors contributed to the drafting of the final version of the paper. HPSS is guarantor.

\section{Funding: None}

Competing interests: International Life Science Institute (ILSI) sponsored TG for travel to Hanoi, Vietnam for the purpose of attending the International Nutritional Anaemia Consultative Group symposium and presenting part of the analysis as a poster.

1 United Nations Administrative Committee on Coordination SubCommittee on Nutrition (ACC/SCN). Fourth report on the world nutrition situation. Geneva: $\mathrm{ACC} / \mathrm{SCN}$ in collaboration with International Food Policy Research Institute, 2000

2 Pollitt E. Iron deficiency anaemia and cognitive function. Ann Rev Nut 1993;13:521-37.

3 American Academy of Pediatrics-Committee on Nutrition. Nutritiona needs of low-birth-weight infants. Pediatrics 1985;75:976-86

4 Hurrell RF. Preventing iron deficiency through food fortification. Nutr Rev 1997;55:210-22.

5 Kochan I. The role of iron in bacterial infections with special consideration of host-tubercle bacillus interaction. Curr Top Microbiol Immuno 1973;60:1-30.

6 Chandra RK. Reduced bactericidal capacity of polymorphs in iron deficiency. Arch Dis Child 1973;48:864-7.

7 Bhaskaram P, Reddy V. Cell mediated immunity in iron and vitamin deficient children. BMJ 1975;iii:522-4.

8 Kaduska MB, Burkitt MJ, Xiang DH, Mason RP. Iron supplementation generates hydroxyl radical in vivo. An ESR spin trapping investigation. J Clin Invest 1995;96:1653-7.
9 Chwang L, Soemantri AG, Polloitt E. Iron supplementation and physica growth of rural Indonesian children. Am J Clin Nutr 1988;47:496-501.

10 Idjradinata P, Watkins WE, Pollitt E. Adverse effect of iron supplementation on weight gain of iron replete young children. Lance 1994:343:1252-4

11 Smith AW, Hendrickse RG, Harrison C, Hayes RJ, Greenwood BM. The effects on malaria of treatment of iron deficiency anaemia with oral iron in Gambian children. Ann Trop Paediatr 1989;9:17-23.

12 Murray MJ, Murray AB, Murray MB, Murray CJ. The adverse effect of iron repletion on the course of certain infections. BMJ 1978;ii:1113-5.

13 Juni P, Altman DG, Egger M. Assessing the quality of randomised controlled trials. In: Egger M, Smith GD, Altman DG, eds. Systematic reviews in health care: meta-analysis in context. London: BMJ Publishing. 2001:87-108

14 Clarke M, Oxman AD, eds. Assessment of study quality. Cochrane reviewers handbook 4.1.1 [updated December 2000]. Cochrane Library. Issue 1. Oxford: Update Software, 2001

15 Sterne JAC, Egger M, Smith GD. Investigating and dealing with publication and other biases. In: Egger M, Smith GD, Altman DG, eds. Systematic reviews in health care: meta-analysis in context. London: BMJ Publishing, 2001:189-208

16 Sterne JAC, Bradburn MJ, Egger M. Meta-analysis in STATA TM. In Egger M, Smith GD, Altman DG, eds. Systematic reviews in health care:metaanalysis in context. London: BMJ Books, 2001:347-69.

17 Deeks JJ, Altman DG, Bradburn MJ. Statistical methods for examining heterogeneity and combining results from several studies in metaanalysis. In: Egger M, Smith GD, Altman DG, eds. Systematic reviews in health care: meta-analysis in context. London: BMJ Books, 2001:285-312.

18 Andelman MB, Bernard RS. Utilization of dietary iron by term infants. Am J Dis Child 1966;111:45-55.

19 Burman D. Haemoglobin levels in normal infants aged 3 to 24 months, and the effect of iron. Arch Dis Child 1972;47:261-71.

20 Damodaran M, Naidu AN, Sarma KVR. Anaemia and morbidity in preschool children. Indian J Med Res 1979;69:448-56.

21 Oppenheimer SJ, Gibson FD, Macfarlane SB, Moody JB, Harrison C, Spencer A, et al. Iron supplementation increases prevalence and effects of malaria: report on clinical studies in Papua New Guinea. Trans $R$ Soc Trop Med Hyg 1986;80:603-12.

22 Bates CJ, Powers HJ, Lamb WH, Gelman W, Webb E. Effect of supplementary vitamins and iron on malaria indices in rural Gambian children. Trans R Soc Trop Med Hyg 1987;81:286-91.

23 Heresi G, Olivares M, Pizarro F, Cayazzo M, Stekel A. Effect of an iron fortified milk on morbidity in infancy: a field trial. Nutr Res 1987;7:915-22.

24 Heywood A, Oppenheimer S, Heywood P, Jolley D. Behavioural effects of iron supplementation in infants in Madang, Papua New Guinea. Am J Clin Nutr 1989;50:630-40.

25 Angeles-Agdeppa I, Schultnik W, Sastroamidjojo S, Gross R, Karyadi D. Weekly micronutrient supplementation to build iron stores in female Indonesian adolescents. Am J Clin Nutr 1997;66:177-83.

26 Heresi G, Pizarro F, Olivares M, Cayazzo M, Hertrampf E, Walter T, et al. Effect of supplementation with an iron fortified milk on incidence of diarrhoea and respiratory infection in urban-resident infants. Scand $J$ Infect Dis 1995;27:385-9.

27 Van Hensbroek MB, Morris-Jones S, Meisner S, Jaffar S, Bayo L, Dackour $\mathrm{R}$, et al. Iron, but not folic acid, combined with effective antimalarial therapy promotes haematological recovery in African children after acute falciparum malaria. Trans R Soc Trop Med Hyg 1995;89:672-6.

28 Beck HP, Felger I, Vounatsou P, Hirt R, Tanner M, Alonso P, et al. Effect of iron supplementation and malaria prophylaxis in infants on Plasmodium falciparum genotypes and multiplicity of infection. Trans $R$ Soc Trop Med Hyg 1999;93:S1/41-S1/45

29 Van Stuijvenberg ME, Kvalsvig JD, Faber M, Kruger M, Kenoyer DG, Benalde AJS. Effect of iron-, iodine-, and $\beta$-carotene-fortified biscuits on the micronutrient status of primary school children: a randomised controlled trial. Am J Clin Nutr 1999;69:497-503.

30 Picaud JC, Rivet C, Salle BL, Basson E, Lasne Y, Chapuis-Cellier C, et al. Early iron supplementation in preterm infants with erythropoietin treatment: Safety and efficacy. Prenat Neonat Med 1999;4:472-8.

31 James JA, Combes M. Iron deficiency in the premature infant: Significance and prevention by intramuscular administration of iron-dextran. Pediatrics 1960;26:368-74.

32 Cantwell RJ. Iron deficiency anaemia of infancy: some clinical principles illustrated by the response of Maori infants to neonatal parenteral iron administration. Clin Pediatr 1972;11:443-9.

33 Fuerth JH. Iron supplementation of the diet in full-term infants: controlled study. J Pediatr 1974;80:974-9.

34 Oppenheimer SJ, Macfarlane SBJ, Moody JB, Bunari O, Hendrickse RG Effect of iron prophylaxis on morbidity due to infectious disease: report on clinical studies in Papua New Guinea. Trans $R$ Soc Trop Med Hy 1986;80:596-602.

35 Harvey PWJ, Heywood PF, Nesheim MC, Galme K, Zegans M, Habicht JP, et al. The effect of iron therapy on malarial infection in Papua New Guinean schoolchildren. Am J Trop Med Hyg 1989;40:12-8.

36 Power HM, Heese HDV, Beatty DW, Hughes J, Dempster WS. Iron fortification of infant milk formula: the effect on iron status and immune function. Ann Trop Paediatr 1991;11:57-66.

37 Javaid N, Haschke F, Pietschnig B, Schuster E, Huemer C, Shebaz A, et al. Interactions between infections, malnutrition and iron nutritional status in Pakistani infants. Acta Paediatr Scand 1991;374:141-50.

38 Irigoyen M, Davidson LL, Carriero D, Seaman C. Randomised, placebocontrolled trial of iron supplementation in infants with low haemoglobin levels fed iron-fortified formula. Pediatrics 1991;88:320-6.

39 Chippaux JP, Schneider D, Aplogan A, Dyck JL, Berger J. Effets de la supplementation en fer sur l'infection palustre. Bull Soc Path Ex 1991;84:54- 
40 Brunser O, Espinoza J, Araya M, Pacheco I, Cruchet S. Chronic iron intake and diarrhoeal disease in infants: a field study in a less-developed country. Eur J Clin Nutr 1993;47:317-26.

41 Angeles IT, Schultnik WJ, Matulessi P, Gross R, Sastroamidjojo S. Decreased rate of stunting among anaemic Indonesian preschool children through iron supplementation. Am J Clin Nutr 1993:58:339-42.

42 Lawless JW, Latham MC, Stephenson LS, Kinoti SN, Pertet AM. Iron supplementation improves appetite and growth in anaemic Kenyan primary school children. J Nutr 1994;124:645-54

43 Hemminki E, Nemet K, Horvath M, Malin M, Schuler D, Hollan S. Impact of iron fortification of milk formulas on infants growth and health. Nutr Res 1995;4:491-503.

44 Van den Hombergh J, Dalderop E, Smit Y. Does iron therapy benefit children with severe malaria-associated anaemia? A clinical trial with 12 weeks supplementation of oral iron in young children from the Turiani division, Tanzania. J Trop Pediatr 1996;42:220-7.

45 Adam Z. Iron supplementation and malaria: a randomised, placebo-controlled field trial in rural Ethiopia. London, University of London, 1996 (PhD thesis).

46 Gebreselassie H. Iron supplementation and malaria infection: Results of a randomised controlled field trial. Quebec: McGill University, $1996(\mathrm{PhD}$ thesis).

47 Mitra AK, Akramuzzaman SM, Fuchs GJ, Rahman MM, Mahalanabis D. Long-term oral supplementation with iron is not harmful for young children in a poor community of Bangladesh. J Nutr 1997;127:1451-5.

48 Palupi L, Schultnik W, Achadi E, Gross R. Effective community intervention to improve haemoglobin status in preschoolers receiving once-weekly iron supplementation. Am J Clin Nutr 1997;65:1057-61.
49 Rosado JL, Lopez P, Munoz E, Martinez H, Allen LH. Zinc supplementation reduced morbidity, but neither zinc nor iron supplementation affected growth or body composition of Mexican preschoolers. Am J Clin Nutr 1997;65:13-9.

50 Menendez C, Kahigwa E, Hirt R, Vounatsou P, Aponte JJ, Font F, et al. Randomised placebo-controlled trial of iron supplementation and malaria chemoprophylaxis for prevention of severe anaemia and malaria in Tanzanian infants. Lancet 1997;350:844-50.

51 Berger J, Dyck JC, Galan P, Aplogan A, Scnheider D, Traissac P, et al. Effect of daily iron supplementation on iron status, cell-mediated immunity, and incidence of infections in 6-36 month old Togolese children. Eur J Clin Nutr 2000;54:29-35.

52 Singhal A, Morley R, Abbott R, Fairweather-Tait S, Stephenson T, Lucas A. Clinical safety of iron fortified formulas. Pediatrics 2000;105:e38.

53 Hallberg L, Ryttinger L, Solvell L. Side effects of oral iron therapy. A double blind study of different iron compounds in tablet form. Acta Med Scand Suppl 1966;459:3-10.

54 Srigiridhar K, Nair KM. Iron deficient intestine is more susceptible to peroxidative damage during iron supplementation in rats. Free Radic Biol Med 1998;25:660-5.

55 Tatli MM, Vural H, Koc A, Kosecik M, Atas A. Altered anti-oxidant status and increased lipid peroxidation in marasmic children. Pediatr Int 2000;42:289-92.

56 Oppenheimer SJ. Iron and its relation to immunity and infectious disease. J Nutr 2001;131(suppl):616-635S.

(Accepted 28 June 2002) 